\title{
3 Research Square

\section{The Application of 3D Printing Technology in the Classification and Preoperative Planning of Complex Tibial Plateau Fractures}

\section{Fuyang Chen}

Nanjing First Hospital

Chenyu Huang

Nanjing First Hospital

Chen Ling

Pukou Branch of Jiangsu Province Hospital

Jinming Zhou

Pukou Hospital

Yufeng Wang

Nanjing First Hospital

Po Zhang

Nanjing First Hospital

Xiao Jiang

Nanjing First Hospital

Xiaoming $\mathrm{Xu}$

Poukou Hospital

Jian Jian

Poukou Hospital

Liming Wang

Nanjing First Hospital

Qingqiang Yao ( $\nabla$ yaoqingqiang@126.com )

Nanjing Medical University https://orcid.org/0000-0002-5037-5412

Research article

Keywords: 3D printing, Tibial plateau fracture, Geometric plane classification, Preoperative planning

Posted Date: August 11th, 2020

DOI: https://doi.org/10.21203/rs.3.rs-29941/v1 
License: (c) (i) This work is licensed under a Creative Commons Attribution 4.0 International License. Read Full License 


\section{Abstract}

Background: Tibial plateau fracture is one of the common intra-articular fractures in clinic. And its accurate classification and treatment is a difficult problem for orthopedic surgeons. Our research aims to investigate the application value of 3D printing in the classification and preoperative planning of complex tibial plateau fractures.

Methods: 28 cases of complex tibial plateau fractures diagnosed and treated in our hospital from January, 2017 to January, 2019.01 were analyzed. Preoperative spiral CT scan was performed and then DICOM data were input into the computer. We use Mimics to process data. And 3D printing technology was applied to print the 3D model of fracture (1:1). Combined with the 3D printed model, the tibial plateau fractures were subdivided into seven types according to the geometric plane of the tibial plateau. The surgical approach was determined on the 3D printed model. And then simulated operations such as accurate reduction of fracture and selection of plate placement were performed.

Results: The reconstructed 3D model of tibial plateau fracture can accurately reflect the direction of fracture displacement and the degree of plateau collapse. Also, it and can help with the preoperative surgical design of tibial plateau fracture. The intraoperative fracture details were basically the same as the 3D printed model. And the fracture surface of the tibial plateau was well improved in all 28 cases.

Conclusion: 3D printing technology can be used to guide the classification and preoperative planning of complex tibial plateau fractures.

\section{Background}

Tibial plateau fracture is one of the common intra-articular fractures in clinic. And its accurate classification and treatment is a difficult problem for orthopedic surgeons[1]. At present, the commonly used clinical classification method of tibial plateau fracture was put forward by Schatzker et al. [2] in 1949 , which is based on X-ray. It does not consider the displacement of fracture in sagittal position. So sometimes the classification can not effectively guide the formulation of treatment plan, especially in the case of posterior tibial plateau. In 2009, Professor Luo Congfeng [3] proposed a three-column classification method of tibial plateau fractures based on $\mathrm{CT}$, which divides the tibial plateau into lateral column region, medial column region and posterior column region. However, this classification method can not directly reflect the degree of comminution and collapse of the articular surface of the fracture. Also, this classification method can not guide the design of preoperative operation well. Complex tibial plateau fractures are often associated with two-column or three-column fractures. Good preoperative planning can greatly shorten the operation time and improve reduction effect. With the emergence of 3D printing digital medical technology, accurate and individualized treatment has become the trend of orthopaedic trauma surgery[4]. 3D printing technology is used to discuss the classification and preoperative planning of complex tibial plateau fractures. 


\section{Methods}

\subsection{Inclusion and exclusion criteria for cases.}

Inclusion criteria: 『cases of unilateral closed tibial plateau fracture treated by surgery in the department of orthopaedics of our hospital during 2017.01-2019.01. $\otimes$ complex tibial plateau fractures with doublecolumn or three-column injuries. $\otimes$ The time from injury to operation was less than 14 days.

Exclusion criteria: \patients with chronic lesions of knee joint and knee joint dysfunction before injury. 『complicated with vascular and nerve injury on the affected side. 邓those with serious underlying diseases or unable to cooperate with treatment.

\subsection{General information.}

A total of 28 patients were included in this study, including 20 males and 8 females, with an average age of $49.5 \pm 2.5$ years old. There were 17 cases of left tibial plateau fracture and 11 cases of right tibial plateau fracture. According to the theoretical basis of three-column classification of tibial plateau,

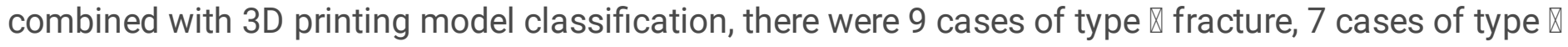
fracture, 4 cases of type $\nabla$ fracture and 8 cases of type $\nabla$ fracture. All cases were treated with internal fixation. The time from injury to operation was 12 days, with an average of $6.5 \pm 1.3$ days. This study has been approved by the Ethics Committee of our hospital. All patients have signed the informed consent form for the operation.

\subsection{Using 3D printing model to refine the classification of plateau fractures from the geometric plane 3-column 3- zone typing method}

3D model printing: all the 28 cases underwent 64 -slice spiral CT thin-slice scanning $(0.6 \mathrm{~mm})$ before operation, and the DICOM data were input into the computer. The Mimics software was used to process the data. And the 3D printing technique was used to print the three-dimensional model of the fracture $(1: 1)$.

Based on the classification of the geometric plane of the platform (figure 1), the overlooking view of the tibial plateau shows that the 0 point is the midpoint of the tibial spine line. The $A^{\prime}$ point is the tibial tubercle. The $B$ ' point is the medial crest of the tibial plateau. And the $C$ 'point is the anterior edge of the fibular head. The tibial plateau is divided into three plane parts by $O A^{\prime}, O^{\prime}$ and $O C^{\prime}$, which are defined as $\mathrm{A}$ zone, $\mathrm{B}$ zone and $\mathrm{C}$ zone respectively.(Table 1) 
Table 1

Three-column and three-zone classification of tibial plateau fractures

\begin{tabular}{|llll|}
\hline Classification & Zone & Position & Approach \\
\hline$\square$ & A & Supine position & Anterolateral approach \\
\hline$\square$ & B & Supine position & Anteriormedial approach \\
\hline$\square$ & C & Prone position & Posterior approach \\
\hline$\square$ & A+B & Supine position & Combined internal and external approach \\
\hline$\square$ & A+C & Supine position & Anterolateral peroneal approach \\
\hline$\square$ & B + C & Supine position & Posteromedial approach \\
\hline$\square$ & A+B + C & Floating position & Anterolateral approach + posteromedial approach \\
\hline
\end{tabular}

Type IV-VII are included in this project

\subsection{Preoperative planning and surgical simulation steps.}

$\nabla$ The CT data of patients with quasi-fracture were processed on a special computer platform. And then the tibial plateau fracture model was printed 1:1 (figure 2). The printing machine is the selective laser sintering equipment Farsoon401. The printing material is nylon powder material for laser sintering.

$\nabla$ The fractures were re-classified by three-column classification according to the fracture imaging data and $3 \mathrm{D}$ visual model so as to achieve accurate classification according to the principle.

Q Evaluate the displacement direction of the bone fracture block. And through the direction, distribution and movement of the fracture block, we can accurately evaluate the displacement direction of the bone fracture block.

$\nabla$ Evaluate the collapse site of the articular surface. And through the detailed analysis and observation of the articular surface, we can determine the actual collapse site of the articular surface.

Q Establish the surgical approach. Through the analysis of the fracture mass and articular surface of the complex tibial plateau fracture, the intraoperative approach can be established to create conditions for reducing trauma injury.

Q Determine the posture and the number of steel plate implantation. Predicting the posture and number of steel plate implantation and the pre-bending data of steel plate before operation can effectively reduce the operation time, trauma injury and the use of anesthetic drugs.

$\nabla$ Simulate the operation. The reduction of the fracture block and the placement of the steel plate can be performed on the 3D model according to the operation plan, so as to improve the proficiency of the 
operation.

\subsection{Preoperative scheme design of complex tibial plateau fracture (type $\mathbb{X}-\mathrm{VII})$.}

Type IV: supine position, choice of surgical approach: combined medial and lateral approach. (figure 3).

Type $\mathbb{~ : ~ s u p i n e ~ p o s i t i o n , ~ c h o i c e ~ o f ~ s u r g i c a l ~ a p p r o a c h : ~ a n t e r o l a t e r a l ~ p e r o n e a l ~ h e a d ~ a p p r o a c h ~ t o ~ f i x ~ t h e ~}$ lateral column, and whether to fix it according to the stability of the posterior column (figure 4).

Type Vl: supine position, choice of surgical approach: modified posterior medial approach (figure 5).

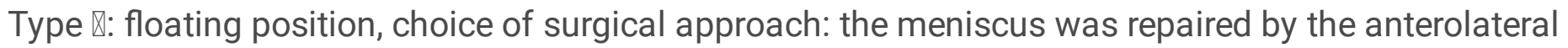
approach, the lateral column was fixed by the anterolateral approach, and the medial column and the posterior column were fixed by the posteromedial approach. (figure 6)

\subsection{Surgical methods and postoperative management.}

All patients were treated with general anesthesia or combined block anesthesia. After successful anesthesia, the affected limb was bound with a tourniquet with a pressure of 50 KPA for 90 minutes. Antibiotics were routinely used before operation. Combined with preoperative classification, the operation was performed according to the surgical approach, fracture reduction mode, plate preshaping, screw direction and length designed before operation. During the operation, the mode and amount of bone graft were determined according to the collapse of the articular surface. Explore the articular surface to repair meniscus and ligament injuries that need one-stage surgical repair as far as possible [5]. Intraoperative Carm fluoroscopy confirmed the degree of fracture reduction and articular surface elevation. After operation, the negative pressure drainage tube was routinely indwelled and removed within 48 hours. Prophylactic use of antibiotics was used within 48 hours after operation. The contraction exercise of quadriceps femoris was performed on the 3rd day after operation. The flexion and extension function of knee joint began to exercise 1 week later and partial weight-bearing began to be carried out 6 weeks after operation. And after the fracture healing was confirmed by X-ray 3 months after operation, patients began to bear weight completely gradually.

\subsection{Observation indicators.}

\subsubsection{The coincidence rate between the preoperative planning and the final surgical plan.}


Each case of complex platform fracture was designed according to the 3D printing model before operation. The specific surgical approach and implant scheme were designed. The specific operation plan was recorded. The consistency rate between the final operation plan and the preoperative plan was obtained.

\subsubsection{Knee joint function score.}

The patients were followed up for 1 year. During the follow-up, the anterior and lateral films of the knee joint were taken and the knee joint function was evaluated by HSS score [6]. HSS score区85 was excellent, 70-84 was good, 60-69 was fair, and score $₫ 59$ was poor.

\subsubsection{Fracture healing time and postoperative complications.}

The fracture healing time of each case was recorded in the follow-up. And whether there were postoperative complications such as infection, screw fracture and other complications were recorded.

\subsection{Statistical processing.}

The above data were analyzed by SPSS18.0 statistical software. $\chi 2$ test was used for the comparison of counting data. $T$ test was used for the comparison of measurement data. The difference was statistically significant (Pष0.05).

\section{Results}

\subsection{The coincidence rate between the preoperative planning and the final surgical plan.}

The classification of tibial plateau fracture combined with 3D printing is basically consistent with what was seen during the operation. The fracture type and articular surface collapse seen during the operation are basically the same as the model, which is of great help to the understanding and operation of the operation. In all 28 cases, the final operation plan of 26 cases was consistent with the design before operation. And 2 cases of them are type VII fracture. Before operation, the lateral column was fixed by anterolateral approach and the medial column and posterior column were fixed by posteromedial approach. The bone mass of lateral column was reduced and fixed by anterolateral approach. It was found that the stability of posterior column was good. The coincidence rate between the preoperative planning and the final surgical plan was $92.8 \%$ (the final plan was consistent with the preoperative planning and design of the number of cases / total number of cases * $100 \%$ ). The postoperative CT results showed that the collapse of the articular surface of the platform was well reduced in all 28 patients. 


\subsection{Knee joint function score.}

During the follow-up 6 months after operation, the excellent and good rate of knee joint function was $85.7 \%$. And the excellent and good rate of knee joint function 12 months after operation was $89.3 \%$. Only one patient had poor knee joint function at 6 months after operation. And his knee joint function was improved after functional rehabilitation exercise.(Table 2)

Table 2

Comparison of knee joint function

\begin{tabular}{|llll|}
\hline & & 6 month after the surgery & 12 month after surgery \\
\hline case numbers & & 28 & 28 \\
\hline HSS score & & $87.3 \pm 6.2$ & $88.1 \pm 7.1$ \\
\multirow{2}{*}{ knee joint function } & Excellent & $19(68 \%)$ & $22(80 \%)$ \\
\cline { 2 - 3 } & Good & $5(18 \%)$ & $3(10 \%)$ \\
\cline { 2 - 3 } & Average & $3(10 \%)$ & $3(10 \%)$ \\
\hline
\end{tabular}

$(\mathrm{P} \otimes 0.05)$

\subsection{Fracture healing time and postoperative complications.}

Postoperative follow-up showed that all 28 patients healed well. And the average healing time was $3.2 \pm 0.6$ months. All 28 patients had no infection and no internal fixation loosening.

\section{Discussion}

\subsection{The importance of correct classification of tibial plateau fractures and preoperative planning}

The treatment of complex tibial plateau fractures has always been a difficult problem in orthopaedics [7]. It is often accompanied by ligament, meniscus injury and serious damage of nerve, blood vessel and soft tissue, which makes the treatment of fracture more difficult [8]. Patients often have varying degrees of dysfunction after operation, which seriously affects their ability to work. How to deal with complex tibial plateau fractures, reduce fracture complications, and make a good recovery of knee joint function is particularly important.

The correct classification and preoperative planning of tibial plateau fractures are very important for the selection of surgical approaches and fixation methods. CT scanning can scan the tibial plateau in axial, 
coronal and sagittal planes to show the full picture of the tibial plateau[13-15]. While 3D printing can have a more intuitive understanding of the whole tibial plateau and the details of the fracture, which makes various measurements more convenient. Thus, we can have a quantitative understanding of the degree of fracture collapse and splitting. Also, it can provide a better reference for surgical approach and internal fixation placement [9].

\subsection{Development of classification methods for tibial plateau fractures}

A good classification method of tibial plateau fracture not only needs to accurately reflect the degree of fracture injury, but also needs to guide clinical treatment. Hohl-Moore classification is a classification method based on X-ray, which divides tibial plateau fractures into 5 kinds of primary fractures and 5 kinds of fracture and dislocation. However, this method can not accurately reflect the degree of soft tissue injury during fracture, and it is difficult to guide clinical treatment. Schatzker classification increases the type of VI fracture with separation of metaphysis and diaphysis, which is widely used in clinic. However, it also has disadvantages. In practical clinical application, simple collapse fracture is rare and can not be effectively distinguished. The fracture of the posterior lateral column of tibial plateau is not distinguished, which is easy to cause missed diagnosis and affect the choice of surgical treatment. The content of $\mathrm{AO}$ classification is detailed, which is conducive to academic research and communication. However, the content is too complex to remember. Also, it can not accurately reflect the relationship between articular surface collapse and fracture severity. Thus, it is not competent to guide the choice of clinical treatment and prognosis [10]. On the basis of the above, Luo Congfeng et al suggested the classical three-column classification method based on three-dimensional CT and analyzed the shape and location of the fracture from a three-dimensional point of view for the first time. It is divided into internal, external and posterior three columns according to the division, which can accurately guide the surgical approach. But this classification did not put forward the concept of posteromedial and posterolateral tibial plateau fractures. Mao Yujiang et al put forward the theory of "four columns and four quadrants" on this basis. This classification distinguishes the morphological differences between posteromedial and posterolateral column fractures, and is of great significance for the guidance of clinical posterior column fractures [12].

With the emergence of 3D printing technology, we can better obtain the solid model of the fracture site before operation. Compared with the solid model, the surgeon has a deeper understanding of the local structure of the fracture. On the basis of accurate classification, it is of greater significance to guide clinical surgery[16-18].

\subsection{Advantages of 3D printing technique in classification and preoperative planning of complex tibial plateau}




\section{fractures}

Compared with CT scanning, 3D printing provides more details about proximal tibial bone and fracture, even the internal details of fracture. 3D printing can intuitively classify and fix the fracture as a more detailed preoperative plan, guide the mode of operation, surgical approach and fixation, which can provide better preoperative guidance for clinical practice. And it can provide the possibility to better improve the classification of tibial plateau fracture. It has the following three advantages:

Psychological advantage: After the 3D model of complex tibial plateau fracture is printed, the operator can intuitively understand the fracture state and displacement. So that the surgeon can have more time and more intuitive to design the operation plan, so as to get twice the result with half the effort.

Operational advantages: According to the operation on the simulated 3D model before operation, the plates and screws can be implanted smoothly during the operation. For medial and lateral plateau fractures, we can often see the articular surface of the plateau, the reduction of the articular surface is not very difficult, but in complex plateau fractures involving posterior column fractures, it may not be easy to see the posterior articular surface during operation. Therefore, the preoperative surgical design, the preshaping of the steel plate and the direction and length of the screw are of great help to the operation. It greatly simplifies the difficulty of the operation, shortens the operation time and reduces the surgical trauma injury[19-20].

Communication advantages: before operation, we use 3D printed models to communicate with patients. So that patients have a better understanding of the severity of their own fractures, as well as a certain understanding of the surgical plan and the expected effect of treatment. In this way, it can also eliminate the patients' fear. And the compliance will be better.

In summary, 3D printing technology can be used to further refine the classification of complex tibial plateau fractures. The surgical approach and internal fixation plan are planned through the solid model, which is intuitive and accurate. And the preoperative planning scheme is feasible, which greatly simplifies the operation and reduces the surgical trauma injury. Also, it can be used as a routine item of preoperative preparation. In the future, under the guidance of 3D printing template, the optimized preoperative planning of tibial plateau fracture and accurate internal fixation operation will be better applied into clinic.

\section{Declarations}

\section{Ethics approval and consent to participate}

All procedures performed in studies involving human participants were in accordance with the ethical standards of the institutional and/or national research committee and with the 1964 Helsinki Declaration and its later amendments or comparable ethical standards. Informed consent was obtained from all individual participants included in the study. 


\section{Consent for publish}

Not applicable.

\section{Availability of data and materials}

The datasets used and/or analyzed during the current study are available from the corresponding author on reasonable request.

\section{Competing interests}

The authors declare that they have no competing interests.

\section{Funding}

National Natural Science Foundation of China(81771985), Key Projects of Medical Science and Technology Development of Nanjing(ZKX18029), Science and Technology Projects of Nanjing(201715073)

\section{Authors' contributions}

$\mathrm{CYH}$ and $\mathrm{CFY}$ contributed to the study design and the drafting of the article. JMZ contributed to the implementation. BZ, HJ and XMX collected CT data and CAD. BJ and FYM performed 3D printing. XJ, FYCand LMW contributed to all surgical procedures. The authors read and approved the final manuscript.

\section{Acknowledgements}

Not applicable.

\section{References}

1. Carrera Ion,Gelber Pablo Eduardo,Chary Gaetan et al. An intact fibula may contribute to allow early weight bearing in surgically treated tibial plateau fractures.[J] .Knee Surg Sports Traumatol Arthrosc, 2018, 26: 756-761.

2. Schatzker j,Mc Broom R,Bruce D. The tibial fracture: the toronto experience 1968-1975. Clin Orthop Relat Res, 1979, 138:94-104.

3. Congfeng Luo, Chengfang Hu, Hong Gao, et al. Three-column classification of tibial plateau fractures

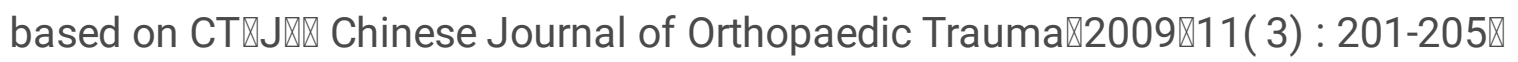


4. Koval KJ,Zuckeman JD. Tibial Plateau. Hand book of fracture. Third edition. 2006:382-387

5. Likun Wang, Zhengkao Sun, Tengbo Yu, et al. Application of three-column theory in the treatment of complex tibial plateau fractures [J]. Chinese Journal of Orthopaedic Trauma, 2014,16(6):495-498.

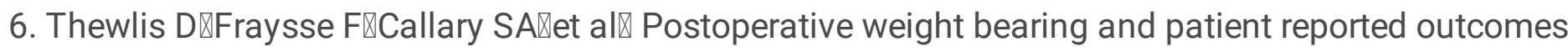

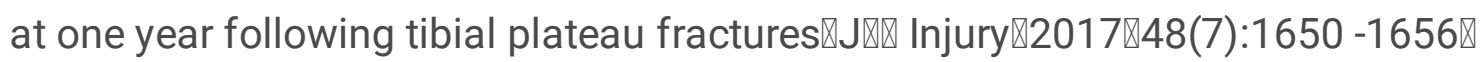

7. Guoqi Wang, Licheng Zhang, Peifu Tang Treatment strategy and progress of tibial plateau fracture $[J]$. Chinese Journal of Orthopaedics $₫ 2016 \rrbracket 36(18): 1202-1207 \rrbracket$

8. Guangsheng Zhang. Study on the effect of bilateral plate internal fixation in the treatment of complex tibial plateau fractures [J]. International Medical and Health Guide ,2017,23(1):62-64.

9. Yucheng Wang, Yunfeng Chen Application of $3 \mathrm{D}$ printing technology in clinical orthopaedics $[\mathrm{J}]$. International Journal of Orthopaedics ,2016,37(6):358-362.

10. Wasserstein $D$,Henry P, Patersong JM,et al.Risk of total knee arthroplasty after operatively treated tibial plateau fracture:amatched-population-based cohort study[J]. Bone Joint Surg Am,2014,96(2):144-150.

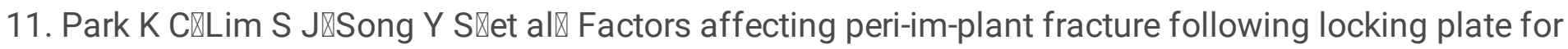
osteoporotic distal femur fractures $\triangle J \mathbb{J} \otimes$ Orthop Traumatol Surg Res $\triangle 2017 \rrbracket 103$ ( 8) : 1201-1204区

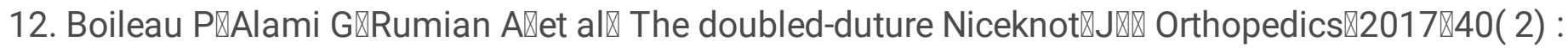
e382 -e386】

13. Gardner MJ, Morshed S, Nork SE, Ricci WM, Chip Routt ML, Jr (2010) Quantification of the upper and second sacral segment safe zones in normal and dysmorphic sacra. J Orthop Trauma 24(10): 622629 ه

14. Mendel T, Noser H, Kuervers J, Goehre F, Hofmann GO, Radetzki F (2013) The influence of sacral morphology on the existence of secure S1 and S2 transverse bone corridors for iliosacroiliac screw fixation. Injury 44(12):1773-1779 》

15. Jeong JH, Jin JW, Kang BY, Jung GH (2017) Can the possibility of transverse iliosacral screw fixation for first sacral segment be pre- dicted preoperatively? Results of a computational cadaveric study. Injury 48(10):2074-207区

16. Beaulé PE, Dorey FJ, Matta JM. Letournel classification for acetabular fractures. Assessment of interobserver and intraobserver reliability. J Bone Joint Surg Am. 2003 Sep;85(9):1704-9.

17. Petrisor BA, Bhandari M, Orr RD, Mandel S, Kwok DC, Schemitsch EH. Improving reliability in the classification of fractures of the acetabulum. Arch Orthop Trauma Surg. 2003 Jun;123(5):228-33. Epub 2003 Apr $\mathbb{8}$

18. Polesello GC, Nunes MA, Azuaga TL, de Queiroz MC, Honda EK, Ono NK. Com- prehension and reproducibility of the Judet and Letournel classification. Acta Ortop Bras. 2012;20(2):70-4.

19. Cunningham BA, Ficco RP, Swafford RE, Nowotarski PJ. Modified iliac oblique- outlet view: a novel radiographic technique for antegrade anterior column screw placement. J Orthop Trauma. 2016 Sep;30(9):e325-30. 
20. Landis JR, Koch GG. The measurement of observer agreement for categorical data. Biometrics. 1977 Mar;33(1):159-74.

\section{Figures}

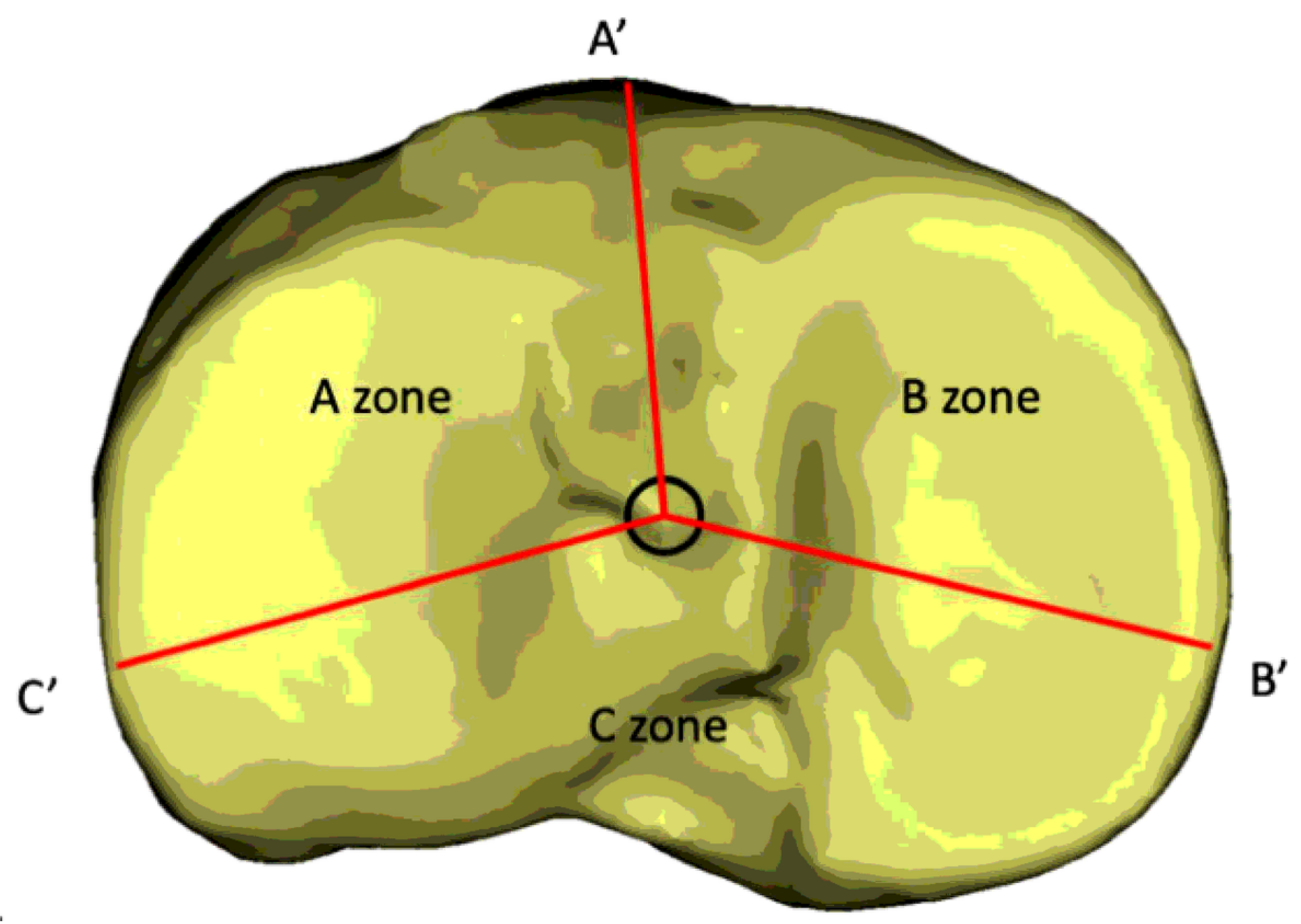

Figure 1

O: Midpoint of tibial spine line A':Tibial Tuberosity B':Medial spine of tibial plateau C': Anterior margin of fibular head 


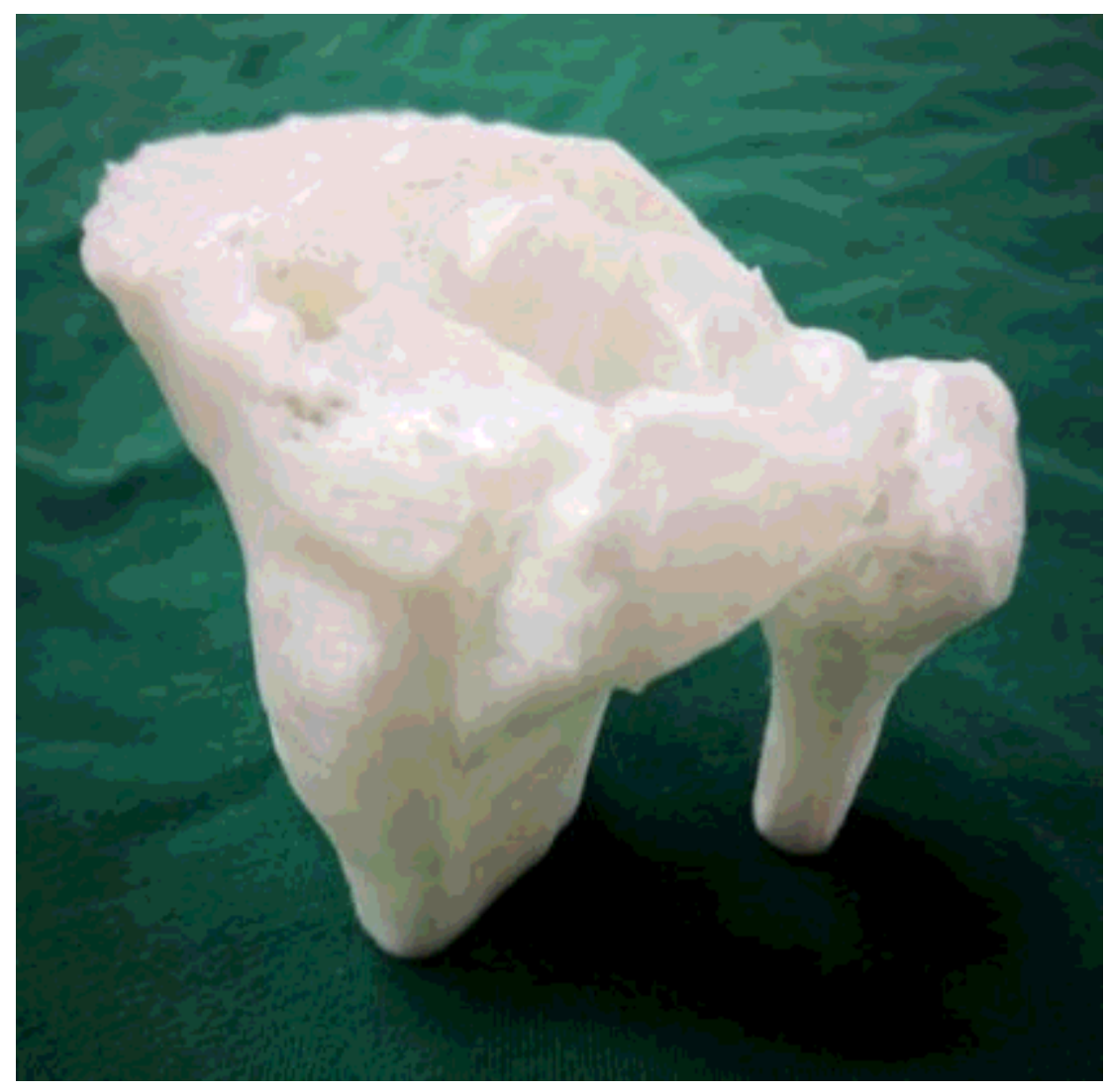

Figure 2

3D printed tibial plateau fracture model
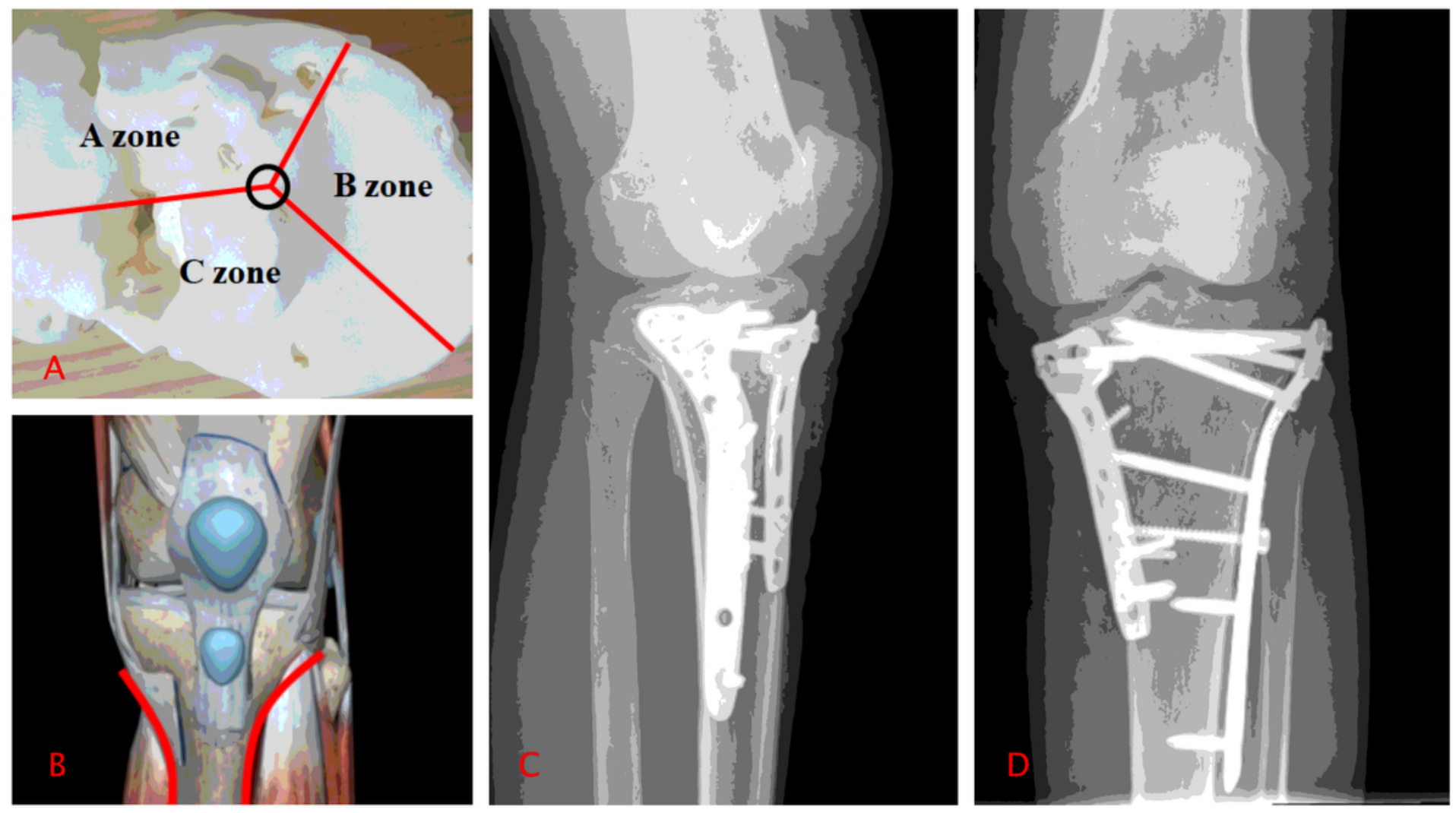

Figure 3 
Type IV tibial plateau fracture A: 3D printed model B: Approach C,D: X-rays after the operation
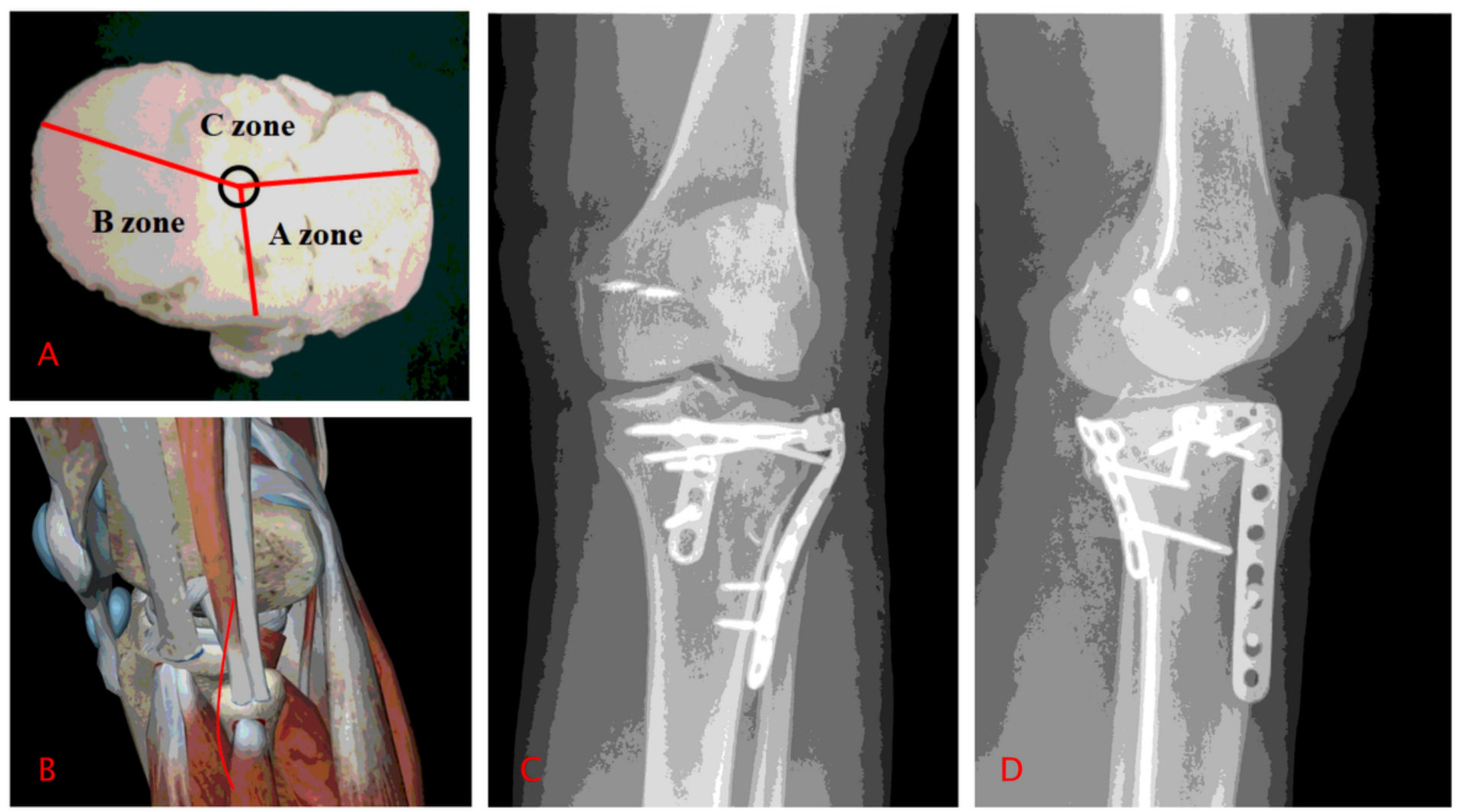

Figure 4

Type V tibial plateau fracture A: 3D printed model B: Approach C,D: X-rays after the operation
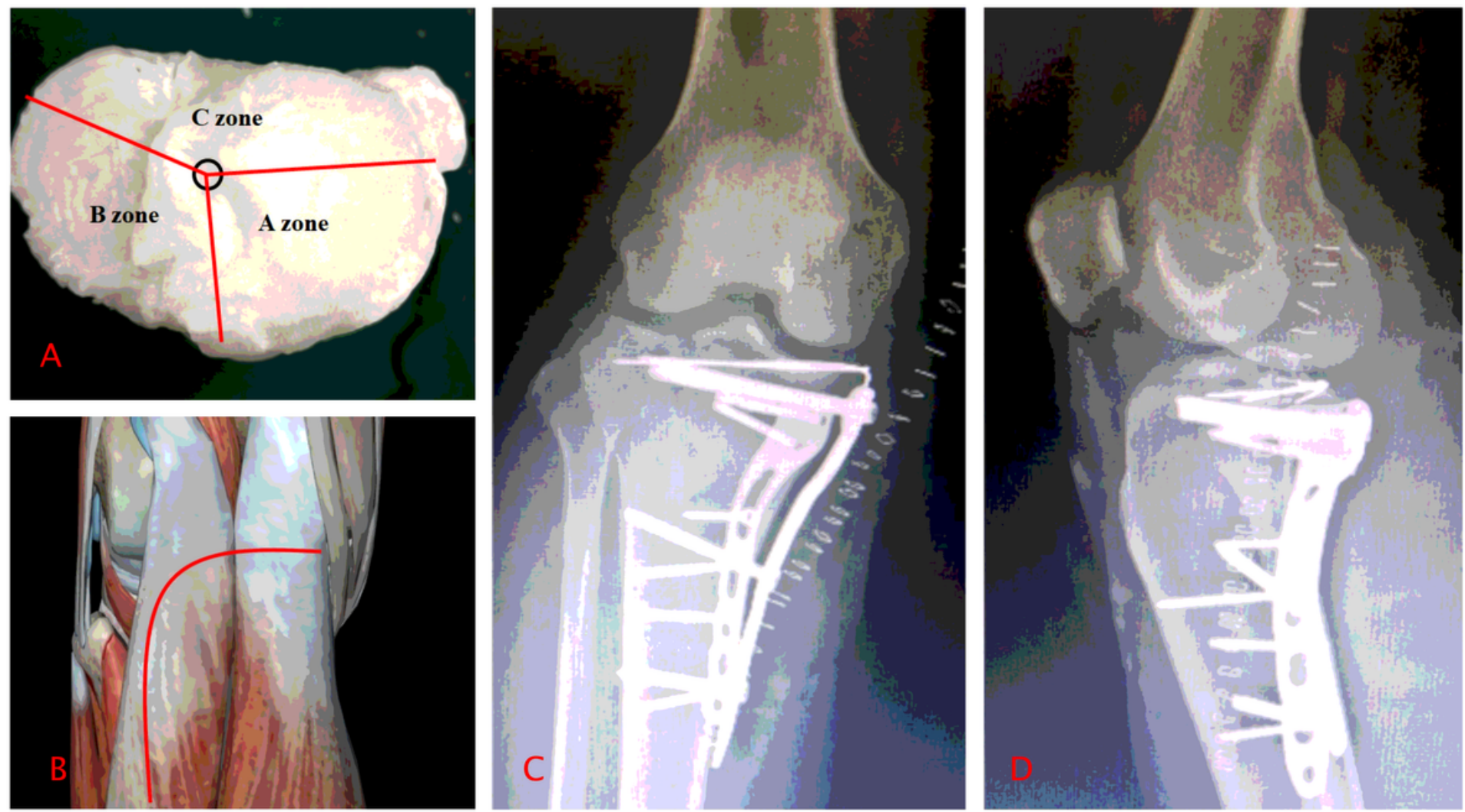
Figure 5

Type VI tibial plateau fracture A: 3D printed model B: Approach C,D: X-rays after the operation
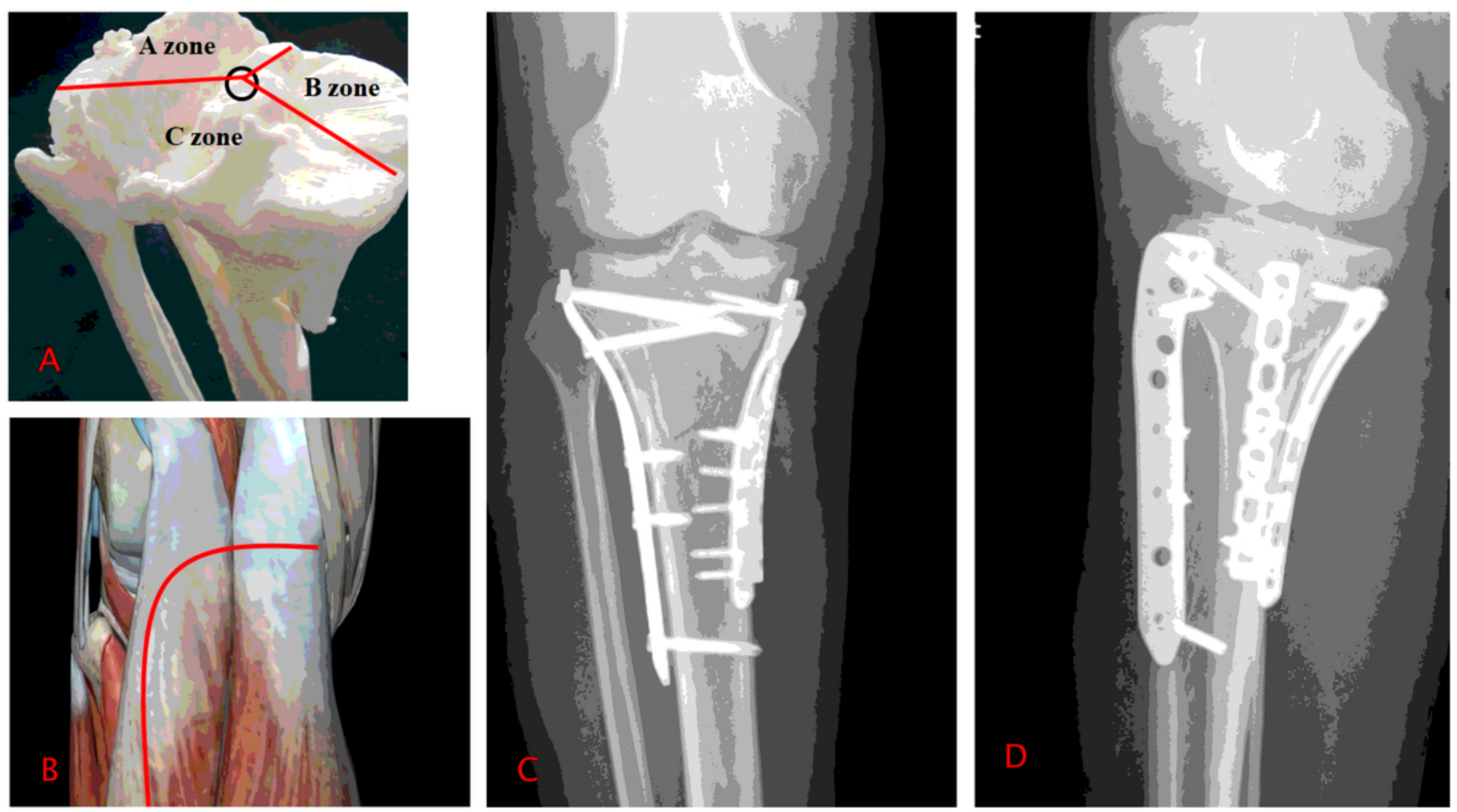

Figure 6

Type VII tibial plateau fracture A: 3D printed model B: Approach C,D: X-rays after the operation 